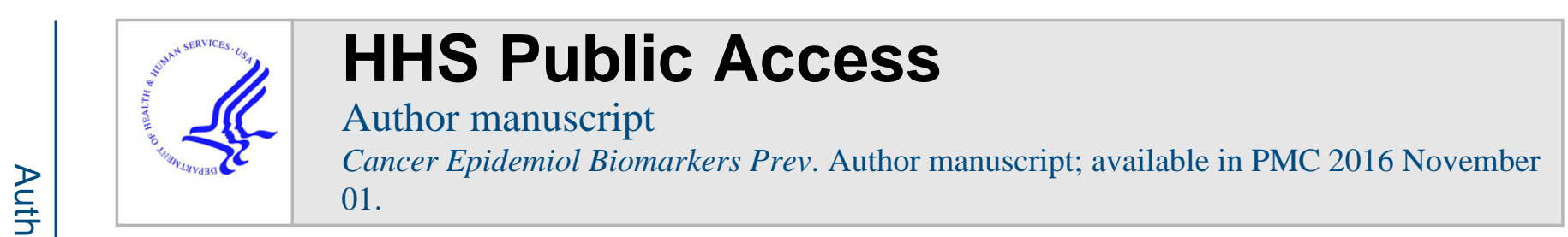

Published in final edited form as:

Cancer Epidemiol Biomarkers Prev. 2015 November ; 24(11): 1789-1795. doi:

10.1158/1055-9965.EPI-15-0327.

\title{
Trajectories of Depressive Symptoms Following Breast Cancer Diagnosis
}

\author{
Nancy E. Avis ${ }^{1}$, Beverly J. Levine ${ }^{1}$, L. Douglas Case ${ }^{2}$, Elizabeth Z. Naftalis ${ }^{3}$, and Kimberly \\ J. Van Zee ${ }^{4}$ \\ ${ }^{1}$ Department of Social Science and Health Policy, Wake Forest School of Medicine, Winston- \\ Salem, North Carolina, USA \\ ${ }^{2}$ Department of Biostatistical Sciences, Wake Forest School of Medicine, Winston-Salem, North \\ Carolina \\ ${ }^{3}$ Baylor University Medical Center, Dallas, Texas \\ ${ }^{4}$ Memorial Sloan-Kettering Cancer Center, New York, New York
}

\begin{abstract}
Background-This longitudinal study sought to identify groups of breast cancer survivors exhibiting distinct trajectories of depressive symptoms up to 24 months following diagnosis, and to describe characteristics associated with these trajectories.

Methods-653 women completed baseline questionnaires within 8 months of breast cancer diagnosis on patient characteristics, symptoms, and psychosocial variables. Depressive symptoms were assessed at baseline and 6,12, and 18 months after baseline. Chart reviews provided cancer and treatment-related data. Finite mixture modeling identified trajectories of depressive symptoms measured with the Beck Depression Inventory (BDI).
\end{abstract}

Results-Six distinct trajectories were identified. Just over half of the sample had consistently very low (3.8\%) or low (47.3\%) BDI scores well below the traditional BDI cut-point of 10 thought to be indicative of clinically significant depression; $29.2 \%$ had consistently borderline scores; $11.3 \%$ had initially high scores that declined over time, but remained above the cut-point; $7.2 \%$ showed increased BDI over time; and a small but unique group (1.1\%) reported chronically high scores above 25 . Women in groups with lower depressive symptom levels were older, had less rigorous chemotherapy, fewer physical symptoms (fatigue and pain), and lower levels of illness intrusiveness.

Conclusions-Approximately $20 \%$ of women had levels of depressive symptoms indicative of clinical depression that were maintained two years post-diagnosis. Factors related to trajectory membership such as illness intrusiveness, social support, fatigue, pain, and vasomotor symptoms suggest targets for possible intervention.

Address correspondence to: Nancy E. Avis, Department of Social Sciences and Health Policy, Division of Public Health Sciences, Wake Forest School of Medicine, Winston-Salem, 27157-1063; navis@ wakehealth.edu; phone: (336) 716-6974; fax:(336) 716-7554. All authors confirm that they have no financial disclosures to report and no conflicts of interest. 
Impact-Results demonstrate the heterogeneity of depressive symptoms following breast cancer and the need for continued screening post treatment.

\section{Keywords}

breast; cancer; depression; survivors; longitudinal

\section{Introduction}

In 2015 an estimated 231,840 American women were diagnosed with breast cancer, the most common cancer among women in the US (1). Depressive symptoms are common among breast cancer patients, with prevalence estimates of persistent depressive symptoms ranging from $12 \%$ to $25 \%$ (2-4). Depressive symptoms have a major impact on quality of life and treatment adherence and may contribute to increased mortality (5-9). For these reasons, ASCO has recently recommended a series of guidelines to better enable clinicians to recognize and treat cancer patients and cancer survivors who show symptoms of depression (10).

The majority of studies on depression among breast cancer patients focus on the first year following diagnosis when risk of depression is greatest $(2,11-16)$. These studies, however, report mean symptom scores according to pre-defined group status (e.g., age group) thus obscuring the heterogeneity that exists among patients. Examination of different patterns of depressive symptoms can provide a more nuanced picture of such heterogeneity and identify women in need (and those not in need) of intervention, thus aiding in better targeting interventions. Bonanno (17) presents evidence that many people show resilience and do not report any psychological symptoms following trauma or loss and proposed four distinct patterns of adjustment to traumatic life events. In addition to a large group of people who show no signs of distress (resilience), other patterns include recovery, delayed distress, and a stable high level of distress. Several studies have grouped cancer patients on the basis of scores above or below a cut-point at more than one time point and find patterns similar to those proposed by Bonanno $(2,18,19)$.

Although providing useful information on patterns of distress and prevalence data on these patterns, the use of predetermined categories may limit what can be learned about underlying heterogeneity and does not allow for examination of latent classes of trajectories. Recent studies conducted among women with breast cancer have used statistical models such as growth mixture modeling to classify women into distinctive trajectories of psychological outcomes, so that there is homogeneity within a trajectory group in scores over time and heterogeneity between groups (12,20-23). Except for Helgeson and colleagues (22) who looked at overall mental health, these studies are limited by their short follow-up period of one year or less. The early years following diagnosis, especially as women transition from active treatment to survivorship, has been identified as a time when distress is likely to occur (24). Previous studies are also limited by inclusion of only a small set of factors examined for their influence on trajectory membership.

The present study uses finite mixture modeling in a larger sample of breast cancer patients ( $\mathrm{N}=653$ ) followed for a longer time than in other studies (up to 24 months post diagnosis). 
The study had two objectives. First we sought to identify groups of women who exhibit different trajectories of depressive symptoms following their breast cancer diagnosis. We hypothesized that there would be varying patterns (trajectories) of depression with some stable and some decreasing. Second, we also examined a large set of predictors, including sociodemographic factors, cancer treatment-related factors, and psychosocial variables, for associations with trajectory group membership. Variables were selected for inclusion in analyses if they were related to depression in our previous analyses and/or in the literature. We hypothesized that age $(3,12,22,25)$, having children under age 18 at home $(2,12,25)$, treatment characteristics $(20,22,25)$, symptoms $(20,23,25)$, illness intrusiveness (25), spirituality $(25,26)$, and social support $(3,25)$ would be related to trajectory group.

\section{Materials and Methods \\ Participants and Procedure}

This longitudinal observational study was conducted among women aged 25 years and older who were newly diagnosed with stage I, II, or III breast cancer. Details have been reported previously (25). Briefly, recruitment was conducted at Memorial Sloan Kettering Cancer Center and the University of Texas - Southwestern Center for Breast Care from 2002-2006; women were followed until 2008. Women were recruited through hospital clinics and advertisements and initially screened by chart review or telephone for eligibility. Eligibility criteria included first-time breast cancer diagnosis, stage I-III at diagnosis, completion of baseline survey within 8 months of diagnosis, at least 18 years of age (although no one in the study was younger than age 25), and ability to read and understand English. Eligible women were mailed a baseline questionnaire to complete and return to the Coordinating Center at Wake Forest University. Follow-up questionnaires were administered 6, 12, and 18 months post completion of the baseline questionnaire (6-26 months post diagnosis). Chart reviews were conducted one year after diagnosis to assess cancer stage and treatment variables.

All sites obtained approval from their Institutional Review Boards.

\section{Measures}

The Beck Depression Inventory (BDI) version BDI-1A (27), a 21-item scale used to assess depressive symptomatology/general distress, was measured on each of the four surveys. The BDI ranges from a possible low of 0 to a possible high of 63 , with a score of 10 and above considered indicative of depression warranting clinical attention (28).

Predictors included patient-related characteristics, cancer-related variables, and psychosocial variables. Patient-related characteristics included age at diagnosis, race (white/non-white), marital/partnered (yes/no), college educated (yes/no), presence of children under age 18 in the home (yes/no), level of difficulty paying for basic necessities (very or somewhat hard vs. not at all hard), and first-degree family history of breast cancer (yes/no) in a female relative (i.e. mother, sister, or daughter). Cancer-related variables, taken from chart reviews, included stage at diagnosis, surgery type (lumpectomy versus mastectomy), chemotherapy regimen (regimen with doxorubicin, regimen without doxorubicin, and no chemotherapy), 
radiation therapy (yes/no), and hormonal therapy (yes/no). Three symptoms previously found associated with depressive symptomatology were included: severity of vasomotor symptoms and fatigue (both measured on a 4-point ordinal scale ranging from none to severe, as used in the Women's Health Initiative [WHI]) (29) and physical pain, measured on a 6-point ordinal scale from the SF-36 (30).

Several psychosocial variables were evaluated for their association with BDI trajectory group membership. Spirituality was measured by the Functional Assessment of Chronic Illness Therapy-Spiritual Well-Being (FACIT-Sp) scale (31), a 12-item scale with two subscales: meaning and peacefulness in one's life and the role of faith. Subscale scores range from 0-32 for meaning and peace (coefficient alpha $=.81$ ) and 0-16 for the role of faith (coefficient alpha $=.88$ ). Higher scores indicate a greater degree of the construct. The Illness Intrusiveness Scale assessed the degree to which breast cancer diagnosis and treatment affected thirteen life areas: health, diet, paid work, active recreation, passive recreation, financial situation, relationship with spouse, sex, family relations, other social relations, selfexpression, religious expression and community (32). Total scores range from 16-112 with higher scores indicating greater intrusiveness (coefficient alpha $=.93$ ). Social Support was assessed by the RAND Social Support Scale (33) which contains 19 items measuring four aspects of support: emotional support, tangible support, affection, and social interaction. The total social support score ranges from 1-5 (coefficient alpha $=.97$ ).

\section{Statistical Analyses}

The group-based SAS finite mixture model procedure PROC TRAJ (34) was applied to identify distinct subgroups of women who followed similar trajectories over time in their BDI scores. This technique identifies distinctive time-based progressions and can model variables with a censored normal distribution (35). The censored normal distribution accurately describes the distribution of scores for the BDI in our sample, where there is a cluster of data at the low end of the scale. The procedure assumes that missing data are missing completely at random (35).

All trajectories were modeled as functions of time since diagnosis which served as a more clinically meaningful metric than time since the baseline survey, which ranged from 1-8 months post diagnosis.

Models were tested that contained from two to seven trajectory groups. We used a combination of a statistical criterion (the Bayesian Information Criterion; BIC) and judgment (i.e., minimum observed group size of $10 \%$ and/or distinctively different trajectories) to select the number of trajectory groups. A higher BIC indicates a better model fit.

Based on the recommendation of Nagin (35), all trajectory groups were modeled including an intercept and a linear and quadratic term for time since diagnosis. The BIC was used as an initial guide in determining the optimal number of trajectory groups within each of the domains. When the BIC pointed to a model where the number of groups appeared higher than optimal because of repetition of similar trajectories, a more parsimonious model with fewer trajectories was chosen. The TRAJ procedure assigns posterior probabilities of group 
membership to all individuals in the data. These probabilities measure a specific individual's likelihood of belonging to each of the model's trajectory groups. For each of the domains investigated here, individuals were assigned to the trajectory group for which they had the maximum posterior probability. For all graphic displays, both the observed mean BDI scores over time are shown for the women assigned to the particular trajectory group as well as the predicted, or expected, trajectory plot line based on the linear and quadratic terms in the trajectory model.

After women were assigned to trajectory groups, associations between group membership and the previously described patient-related, cancer-related, and psychosocial covariates were assessed using chi-square tests (for categorical variables) and F-tests (for continuous variables). For the continuous symptom and psychosocial measures, we assessed trajectory group differences in the estimated values at four months (the median time from diagnosis to baseline). PROC MIXED was used to model the repeated longitudinal measures of these variables using time (months since diagnosis), time squared, and trajectory group membership as independent variables. Estimated means for each of these variables at 4 months were obtained using "estimate" statements for each of the trajectory groups.

All analyses were carried out using SAS version 9.3.

\section{Results}

\section{Participants}

A total of 740 surveys were mailed out to women deemed eligible from chart reviews or telephone screening; 653 of these women completed baseline surveys and were determined eligible for an initial response rate of $88 \%$. A total of 565 women remained in the study and completed all four surveys for a retention rate of $86.5 \%$. Sample characteristics are shown in Table 1 . The sample was predominantly Caucasian, well-educated women who were diagnosed with stage I or stage II disease and who had a mean age close to 55. The average time between diagnosis and administration of the baseline survey across all the women in the sample was 4.5 months (range $=3$ days to 7.4 months). There were no significant differences by trajectory group in mean time since diagnosis.

\section{Model Selection}

Table 2 displays the BIC for different trajectory models, each model corresponding to a differing number of allowed trajectory groups, ranging from two to seven, along with the estimated percentages of the sample that fell into the trajectory groups resulting from the model. These estimated percentages are based on sums of posterior probabilities, and thus differ slightly from the percentages reported earlier, and from those reported in Figure 1, which are based on the actual number of women assigned to the groups. Although the largest BIC (i.e., the smallest in absolute value of the negative BICs) of any of the models was associated with the seven-trajectory model, the increase in information (i.e., the change in BIC) from six to seven trajectories was miniscule. The change in BIC for all the previous models was comparatively large. The six-trajectory model allowed for greater refinement of the different groups who had higher levels of BDI over time compared to the refinement 
allowed by the two immediately smaller models. An increase to seven trajectories did not appear to add any new information over the six trajectory model.

The posterior probabilities associated with group assignment in the six-trajectory model ranged from 0.37 to 1.0 , with $76 \%$ of all participants having their posterior probability of group assignment above 0.75 . Fifty-three percent of the sample had a posterior probability above 0.90 .

Using a combination of information from the BIC and observation of the distinctiveness of trajectories, the six-trajectory model was selected as the best model to describe the data, even though the sixth trajectory group, comprised of those with consistently high levels of BDI, was made up of only $1.1 \%(n=7)$ of the entire sample. Because this group was so small, we do not provide descriptive data on it below, nor conduct inferential statistical analyses on it. We also did not combine these women with those of any other trajectory, because of their unusually high BDI levels.

\section{Description of Depression Trajectories}

As seen in Figure 1, slightly more than half the sample ( $\mathrm{n}=334,51.1 \%)$ fell into Groups 1 and 2, both of which had consistently low levels of depressive symptoms over time, with Group 1 essentially not reporting any depressive symptoms. Almost a third of the sample ( $\mathrm{n}=191,29.2 \%$ ) fell into Group 3, which had a consistently borderline level of depressive symptoms. Their BDI score hovered around 10, which is cited by some as indicative of clinically significant depression(28). Women in group $4(n=74,11.3 \%)$ showed some decline in depressive symptoms over the first year, but still had a BDI score above 10 at 24 months. Group $5(\mathrm{n}=47,7.2 \%)$ showed a rise in depressive symptoms around 10 months post diagnosis that remained fairly high. Finally, as noted above, a small group of women $(\mathrm{n}=7,1.1 \%)$ in Group 6 reported consistently high BDI scores (around 30$)$.

\section{Associations with Trajectory Group Membership}

Table 3 presents characteristics of each trajectory group (with the exception of Group 6), along with corresponding significance test results and p-values. Table 4 shows the estimated means (at 4 months post-diagnosis) of symptoms and psychosocial measures for each group. There were significant differences across groups in many of these measures, with clear general profiles emerging. Levels of many characteristics paralleled levels of BDI. Of all groups, the steady very low and low groups (Groups 1 and 2) were most similar in profiles.

Relative to all other groups, women in Group 1 were the oldest and had the most favorable cancer-related characteristics (i.e., early stage at diagnosis, lumpectomy). They were the least likely to report receiving hormonal therapy, and, along with group 2, were least likely to report chemotherapy. They were also the least likely to report difficulty paying for basics and most likely to be non-Hispanic white. They reported lowest levels of physical symptoms (vasomotor, pain, and fatigue) and illness intrusiveness, and highest levels of spiritualitymeaning/peace and social support.

Women in Group 2 were the second oldest group and were the second most likely of all the groups to be diagnosed at stage I and to have a lumpectomy. They reported low levels of 
symptoms and illness intrusiveness scores and high levels of meaning/peace and social support. Along with Group 1, they were least likely to report chemotherapy. However, they were the most likely to report hormonal therapy.

The characteristics of women in Group 3 were generally intermediate between those of Groups 1 and 2 and Groups 4 and 5, in terms of stage at diagnosis, receipt of chemotherapy, type of surgery, and measures of symptoms and illness intrusiveness. Women in Group 3 were second most likely, after group 2, to report receiving hormonal therapy.

Women in Group 4 started out with a high BDI and experienced some decline over time. They were the youngest women on average, and, along with women in Group 5, were most likely to report having children under age 18 at home and experiencing vasomotor symptoms. Women in Group 4 were also the most likely to have cancer-related difficulties: they were likely to be diagnosed at a later stage and most likely to have chemotherapy with doxorubicin, to have had a mastectomy, and had the highest level of illness intrusiveness.

Group 5 showed high levels of depressive symptoms throughout the 24 months, and even showed a slight increase over time. The women in group 5 were the most likely to be nonwhite and to report difficulty paying for basics. They were second to Group 1 in being unlikely to receive hormonal therapy. They also reported the lowest levels of social support.

\section{Discussion}

Consistent with our initial hypothesis, multiple distinct patterns of depressive symptoms emerged in our sample of breast cancer survivors. Just over half of the sample reported consistently low levels of depressive symptoms over time and the other half showed a variety of different trajectories. This trajectory analysis goes beyond conventional analyses that examine only mean levels of depression in pre-determined strata to present a more detailed portrait of women displaying different response patterns, including a group of women for whom depressive symptoms do not decline.

There are some consistencies between our results and those of other studies, especially in our finding a relatively large proportion of women with few or no depressive symptoms following diagnosis $(12,20,21)$. Combining the very low and low groups, $51 \%$ of our sample was well below the BDI cut point of 10 for depression throughout the 26 months post-diagnosis. Among 172 breast cancer survivors followed from diagnosis to up to 6 months post treatment, Henselmans (21) found that $46 \%$ of their sample reported no distress on the General Health Questionnaire and another $33 \%$ reported distress only during active treatment. In their sample of 398 women assessed prior to surgery and 6 months after surgery, Dunn and colleagues (12) found that $39 \%$ of women were well below the cut-point on the CES-D in the 6 months following surgery. A study of 285 Chinese women (20) found that $66 \%$ of women were in a resilient group on the Chinese Health Questionnaire over an 8month post-surgery period. Although specific percentages differ, all of these suggest a relatively large group of women who do not show signs of depressive symptoms following a breast cancer diagnosis. Such results support the call for psychosocial interventions to be targeted towards those in need, rather than promoted to all women (36). 
However, perhaps due to our larger sample size and resulting greater statistical power, we also found more distinct trajectories than previous studies (12, 20-23). Importantly, we also found close to $20 \%$ of women who still had elevated depressive symptoms at least 24 months post diagnosis and who may be in need of intervention. Although comparisons to other studies are difficult due to variations in measures and samples, the percentage of women in the present study with depressive symptoms is higher than figures reported from an NHANES general population study (37), where $9.8 \%$ of the sample aged $40-59$ and $5.4 \%$ of those 60 and older were found to have moderate to severe depressive symptoms.

However, the percentage of women in our sample who report depressive symptoms is less than figures reported among other patient groups. Studies using the BDI found that $51 \%$ of congestive heart failure patients (38), $46 \%$ of patients seeking reconstructive services following burn injuries (39), and 51-65\% of patients with systemic sclerosis had BDI scores $>10$ (40). Finally, $31 \%$ (computed as a weighted average across studies) of patients hospitalized following myocardial infarction were reported to have BDI scores >10 (41).

Although previous studies of depression trajectories have identified a group of women that recover over time $(12,20-23)$ our data would suggest that this recovery may hit a plateau for some. We also found a group that showed an increase in depression that has not been found by others. These findings reinforce recommendations that screening for depression continue from the point of diagnosis onward (10).

The present analyses found a range of variables that distinguished the trajectory groups: age at diagnosis, difficulty paying for basics, race, having children under age 18 at home, cancer stage, chemotherapy, hormonal therapy, symptoms, spirituality, social support, and illness intrusiveness. Except for chemotherapy, cancer stage, and having children under 18 at home these variables were either not included in other trajectory studies or did not significantly distinguish trajectory groups that had a more limited range of covariates and smaller sample size, although they have been found by others to be related to depression $(2,3,12,20,22$, $23,25,26)$. The present trajectory analysis goes beyond previous analyses in several ways. First, they uncover important new patterns of heterogeneity in level of depressive symptomatology among women following a breast cancer diagnosis. Second, the current analyses aid in identifying early levels of factors (e.g., high levels of illness intrusiveness and symptoms) that appear to be predictive of specific trajectories of continued depressive symptoms. Of additional note, we found that the distinction between chemotherapy with and without doxorubicin is an important one that previous studies have not made.

There are several limitations of the present study. First, we may have underestimated the percentage of women who maintain high levels of depressive symptoms. As has been recognized in other longitudinal studies $(12,21)$, women who do not remain in a study may be having more difficulty than those who complete all surveys. Women who dropped out of our study had a significantly higher mean BDI level at baseline (mean $=10.1)$ than those women who remained in the study for at least two of the four surveys (mean = 8.1) and may have continued to have high levels of depressive symptoms. They were also likely to be younger. We conducted a sensitivity analysis to examine whether the women who did not have BDI data at all 4 time points exerted a meaningful influence on our trajectory analysis. When we limited our trajectory analysis to only women with complete data, we obtained the 
same 6-group optimal solution (according to the BIC), and each of the six groups contained close to the same percentage of the sample as they did in our reported analyses. We are thus confident that missing data did not unduly influence our findings or our conclusions about major trajectory patterns. Second, the Beck Depression Inventory is a self-report measure of depressive symptomatology and not a measure of clinical depression. Further, although characteristic of many samples of breast cancer patients, this sample is relatively homogeneous (mostly white and educated) which limits the generalizability of our findings. Another limitation is that the maximum follow-up was 26 months post-diagnosis. Finally, a pre-diagnosis measure of depression (or at least a history of depression) may provide additional insight into the women comprising the different trajectory groups and especially group 6, the group that had very high depressive symptoms that we were unable to analyze statistically because of small size. Women who fall into such a group may be the most in need of clinical intervention.

Strengths of this trajectory analysis over previous trajectory analyses include larger sample size, longer time period covered, and a wider range of predictors. This study has several implications for both research and clinical care. The use of finite mixture modeling provides insight into the heterogeneity of women's depressive symptoms following breast cancer; traditional analyses rely on averages taken across such latent heterogeneity. This analytic approach could be applied to other symptoms or outcomes to help identify specific groups of patients most in need of intervention. Some factors related to trajectory membership such as illness intrusiveness, social support, fatigue, pain, and vasomotor symptoms suggest targets for possible intervention.

These results also have implications for clinical care. Approximately $20 \%$ of women had levels of depressive symptoms indicative of clinical depression and another $29 \%$ had borderline levels that were maintained two years post-diagnosis, thus indicating the need for continued screening. These results clearly support the ASCO recommendations for continued screening for depression even after the end of treatment (10). Untreated depression is a significant problem that has implications for quality of life, treatment adherence and mortality (5-9).

\section{Acknowledgments}

The authors thank the women who participated in the study and the study staff.

Financial Support. This study was supported by a grant \# DAMD17-01-1-0447 from the Department of Defense (N.E. Avis, L.D. Case, E.Z. Naftalis, K.J. Zee) and Grant P30 CA012197 from the National Cancer Institute (L.D. Case). The content is solely the responsibility of the authors and does not necessarily represent the official views of the National Institutes of Health or the Department of Defense.

\section{References}

1. American Cancer Society. Cancer Facts \& Figures 2014. Vol. 2014. Atlanta: American Cancer Society; 2014.

2. Deshields T, Tibbs T, Fan M, Taylor M. Differences in patterns of depression after treatment for breast cancer. Psychooncology. 2006; 15:398-406. [PubMed: 16100708] 
3. Burgess C, Cornelius V, Love S, Graham J, Richards M, Ramirez A. Depression and anxiety in women with early breast cancer: five year observational cohort study. BMJ. 2005; 330:702. [PubMed: 15695497]

4. Krebber AM, Buffart LM, Kleijn G, Riepma IC, de BR, Leemans CR, et al. Prevalence of depression in cancer patients: a meta-analysis of diagnostic interviews and self-report instruments. Psychooncology. 2014; 23:121-30. [PubMed: 24105788]

5. Bower JE. Behavioral symptoms in patients with breast cancer and survivors. J Clin Oncol. 2008; 26:768-77. [PubMed: 18258985]

6. Day R, Ganz PA, Costantino JP. Tamoxifen and depression: more evidence from the National Surgical Adjuvant Breast and Bowel Project's Breast Cancer Prevention randomized study. J Natl Cancer Inst. 2001; 93:1615-23. [PubMed: 11698565]

7. DiMatteo MR, Lepper HS, Croghan TW. Depression is a risk factor for noncompliance with medical treatmentMeta-analysis of the effects of anxiety and depression on patient adherence. Archives of Internal Medicine. 2000; 160:2101-7. [PubMed: 10904452]

8. Fann JR, Thomas-Rich AM, Katon WJ, Cowley D, Pepping M, McGregor BA, et al. Major depression after breast cancer: a review of epidemiology and treatment. Gen Hosp Psychiat. 2008; 30:112-26.

9. Mols F, Husson O, Roukema JA, Poll-Franse L. Depressive symptoms are a risk factor for all-cause mortality: results from a prospective population-based study among 3,080 cancer survivors from the PROFILES registry. J Cancer Surviv. 2013; 73:484-92. [PubMed: 23677523]

10. Andersen BL, DeRubeis RJ, Berman BS, Gruman J, Champion VL, Massie MJ, et al. Screening, assessment, and care of anxiety and depressive symptoms in adults with cancer: an American Society of Clinical Oncology Guideline adaptation. J Clin Oncol. 2014; 32:1605-19. [PubMed: 24733793]

11. Compas BE, Stoll MF, Thomsen AH, Oppedisano G, Epping-Jordan JE, Krag DN. Adjustment to breast cancer: age-related differences in coping and emotional distress. Breast Cancer Res Tr. 1999; 54:195-203.

12. Dunn LB, Cooper BA, Neuhaus J, West C, Paul S, Aouizerat B, et al. Identification of distinct depressive symptom trajectories in women following surgery for breast cancer. Health Psychol. 2011; 30:683-92. [PubMed: 21728421]

13. McCorry NK, Dempster M, Quinn J, Hogg A, Newell J, Moore M, et al. Illness perception clusters at diagnosis predict psychological distress among women with breast cancer at 6 months post diagnosis. Psychooncology. 2012; 22:692-8. [PubMed: 22389291]

14. Somerset W, Stout SC, Miller AH, Musselman D. Breast cancer and depression. Oncology. 2004; 18:1021-34. [PubMed: 15328896]

15. Wenzel LB, Fairclough DL, Brady MJ, Cella D, Garrett KM, Kluhsman BC, et al. Age-related differences in the quality of life of breast carcinoma patients after treatment. Cancer. 1999; 86:1768-74. [PubMed: 10547550]

16. Wong-Kim EC, Bloom JR. Depression experienced by young women newly diagnosed with breast cancer. Psychooncology. 2005; 14:564-73. [PubMed: 15543537]

17. Bonanno GA. Loss, trauma, and human resilience: have we underestimated the human capacity to thrive after extremely aversive events? Am Psychol. 2004; 59:20-8. [PubMed: 14736317]

18. Gallagher J, Parle M, Cairns D. Appraisal and psychological distress six months after diagnosis of breast cancer. Br J Health Psychol. 2002; 7:365-76. [PubMed: 12614506]

19. Millar K, Purushotham AD, McLatchie E, George WD, Murray GD. A 1-year prospective study of individual variation in distress, and illness perceptions, after treatment for breast cancer. $\mathrm{J}$ Psychosom Res. 2005; 58:335-42. [PubMed: 15992569]

20. Lam WW, Bonanno GA, Mancini AD, Ho S, Chan M, Hung WK, et al. Trajectories of psychological distress among Chinese women diagnosed with breast cancer. Psychooncology. 2010; 19:1044-51. [PubMed: 20014074]

21. Henselmans I, Helgeson VS, Seltman H, de Vries J, Sanderman R, Ranchor AV. Identification and prediction of distress trajectories in the first year after a breast cancer diagnosis. Health Psychol. 2010; 29:160-8. [PubMed: 20230089] 
22. Helgeson VS, Snyder P, Seltman H. Psychological and physical adjustment to breast cancer over 4 years: identifying distinct trajectories of change. Health Psychol. 2004; 23:3-15. [PubMed: 14756598]

23. Donovan KA, Gonzalez BD, Small BJ, Andrykowski MA, Jacobsen PB. Depressive symptom trajectories during and after adjuvant treatment for breast cancer. Ann Behav Med. 2014; 47:292302. [PubMed: 24158626]

24. Hewitt, ME.; Greenfield, S.; Stovall, E. From cancer patient to cancer survivor: lost in transition. Washington, D.C.: National Academy Press; 2006.

25. Avis NE, Levine B, Naughton MJ, Case LD, Naftalis E, Van Zee KJ. Age-related longitudinal changes in depressive symptoms following breast cancer diagnosis and treatment. Breast Cancer Res Treat. 2013; 139:199-206. [PubMed: 23588951]

26. Yanez B, Edmondson D, Stanton AL, Park CL, Kwan L, Ganz PA, et al. Facets of spirituality as predictors of adjustment to cancer: relative contributions of having faith and finding meaning. $\mathrm{J}$ Consult Clin Psychol. 2009; 77:730-41. [PubMed: 19634965]

27. Beck AT, Steer RA, Ball R, Ranieri W. Comparison of Beck Depression Inventories -IA and -II in psychiatric outpatients. J Pers Assess. 1996; 67:588-97. [PubMed: 8991972]

28. Beck, AT.; Steer, RA. Beck Depression Inventory Manual. San Antonio: Harcourt Brace Jovanovich; 1987.

29. Barnabei VM, Cochrane BB, Aragaki AK, Nygaard I, Williams RS, McGovern PG, et al. Menopausal symptoms and treatment-related effects of estrogen and progestin in the Women's Health Initiative. Obstet Gynecol. 2005; 105:1063-73. [PubMed: 15863546]

30. Ware JE Jr, Sherbourne CD. The MOS 36-item short form health survey (SF-36): I. Conceptual framework and item selection. Med Care. 1992; 30:473-83. [PubMed: 1593914]

31. Peterman AH, Fichett G, Brady MJ, et al. Measuring spiritual well-being in people with cancer. The Functional Assessment of Chronic Illness Therapy-Spiritual Well-Being Scale (FACIT-sp). Ann Behav Med. 2002; 24:49-58. [PubMed: 12008794]

32. Devins GM. Using the Illness Intrusiveness Ratings Scale to understand health-related quality of life in chronic disease. J Psychsom Res. 2010; 68:591-602.

33. Sherbourne CD, Stewart AL. The MOS Social Support Survey. Soc Sci Med. 1991; 32:705-14. [PubMed: 2035047]

34. Jones, BL. Traj: Group-based modeling of longitudinal data. 2010. Retrieved from: http:// www.andrew.cmu.edu/user/bjones/index.htm. Ref Type: Online Source

35. Nagin, DS. Group-Based Modeling of Development. Cambridge, MA: Harvard University Press; 2005.

36. Jacobsen, PB.; Donovan, KA.; Swaine, ZN. Management of anxiety and depression in adult cancer patients: Toward an evidence-based approach. In: Chang, AE.; Ganz, PA.; Hayes, DF., editors. Oncology: An Evidence-Based Approach. Philadelphia, PA: Springer; 2006. p. 1552-79.

37. Pratt, LA. Depression in the U.S. Household Population, 2009-2012. 2015. Retreived from: http:// www.cdc.gov/nchs/data/databriefs/db172.pdf. Ref Type: Online Source

38. Freedland KE, Rich MW, Skala JA, Carney RM, Davila-Roman VG, Jaffe AS. Prevalence of depression in hospitalized patients with congestive heart failure. Psychosom Med. 2003; 65:119_ 28. [PubMed: 12554823]

39. Thombs BD, Haines JM, Bresnick MG, Magyar-Russell G, Fauerbach JA, Spence RJ. Depression in burn reconstruction patients: symptom prevalence and association with body image dissatisfaction and physical function. Gen Hosp Psychiatry. 2007; 29:14-20. [PubMed: 17189739]

40. Thombs BD, Taillefer SS, Hudson M, Baron M. Depression in patients with systemic sclerosis: a systematic review of the evidence. Arthritis Rheum. 2007; 57:1089-97. [PubMed: 17665491]

41. Thombs BD, Bass EB, Ford DE, Stewart KJ, Tsilidis KK, Patel U, et al. Prevalence of depression in survivors of acute myocardial infarction. J Gen Intern Med. 2006; 21:30-8. [PubMed: 16423120] 


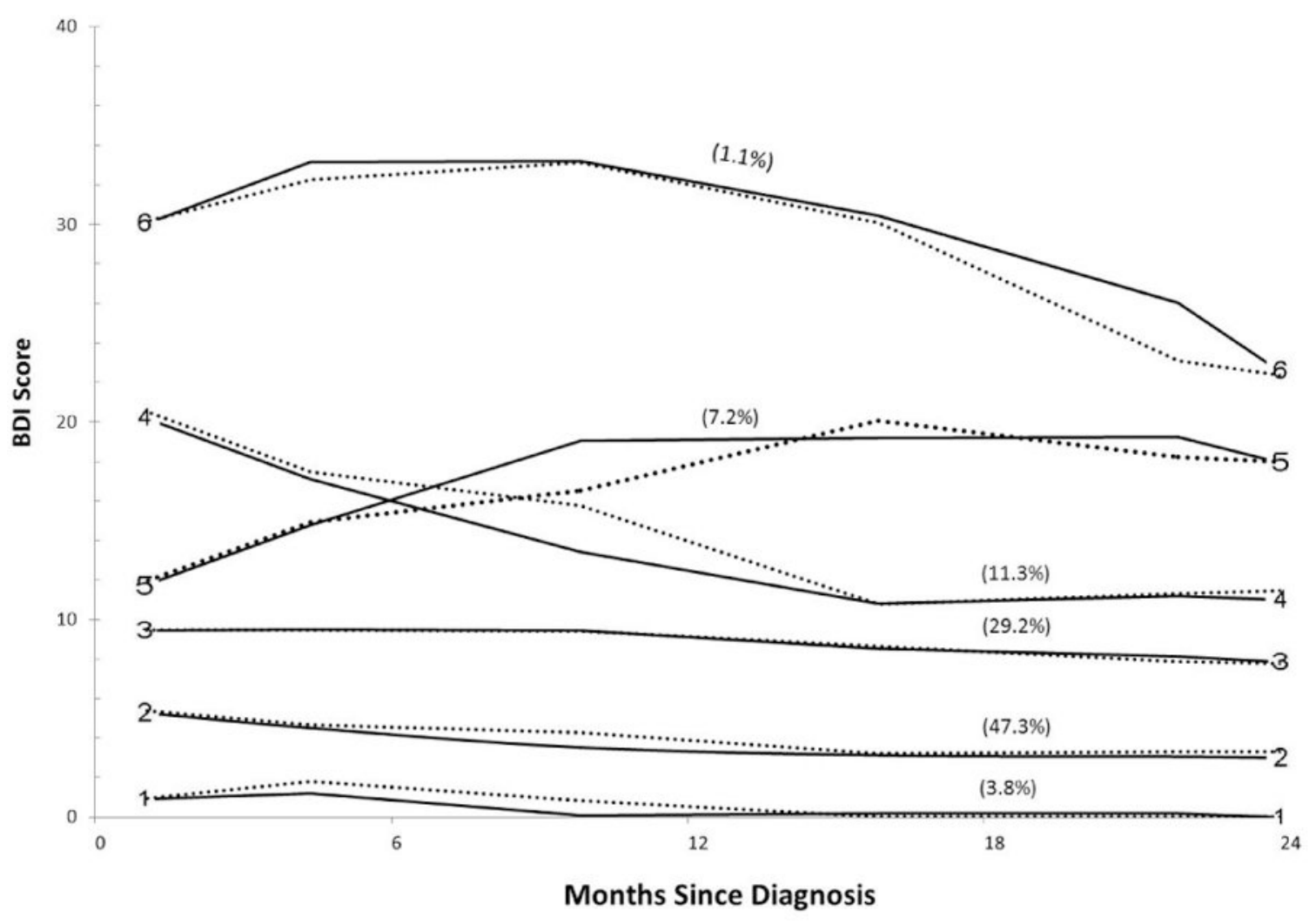

Figure 1.

Predicted (dashed lines) and observed (solid lines) levels of depressive symptoms for each trajectory group by months since diagnosis. Scores of 10 and above are indicative of clinically significant depression. Percentages refer to the percent of participants in each trajectory group. 
Table 1

Characteristics of the Study Sample $(\mathrm{N}=653)$

\begin{tabular}{lcc}
\hline Characteristic & n & $(\%)$ \\
\hline Sociodemographic variables & & \\
Race (White) & 585 & $(90)$ \\
Married/partnered & 468 & $(72)$ \\
College Educated & 409 & $(63)$ \\
Children <18 in home & 171 & $(26)$ \\
First degree family history & 135 & $(21)$ \\
Cancer-related & & \\
Cancer stage & & \\
$\quad$ I & 338 & $(52)$ \\
$\quad$ II & 262 & $(40)$ \\
$\quad$ III & 53 & $(8)$ \\
Lumpectomy & 415 & $(64)$ \\
Radiation therapy & 472 & $(72)$ \\
Chemotherapy & 436 & $(67)$ \\
$\quad$ No Chemotherapy & 216 & $(33)$ \\
Chemotherapy with doxorubicin & 327 & $(50)$ \\
Chemotherapy, no doxorubicin & 110 & $(17)$ \\
\hline Hormonal therapy & 476 & $(73)$ \\
\hline & Mean & \\
\hline Age at diagnosis, y & $54.9 \pm 0.5$ \\
Time since diagnosis at study entry, mo & $4.5 \pm 0.05$ \\
\hline
\end{tabular}

Abbrevations: SE, standard error of sample mean 


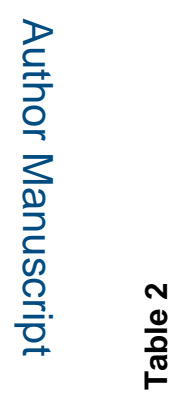

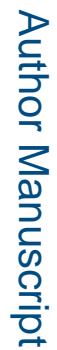

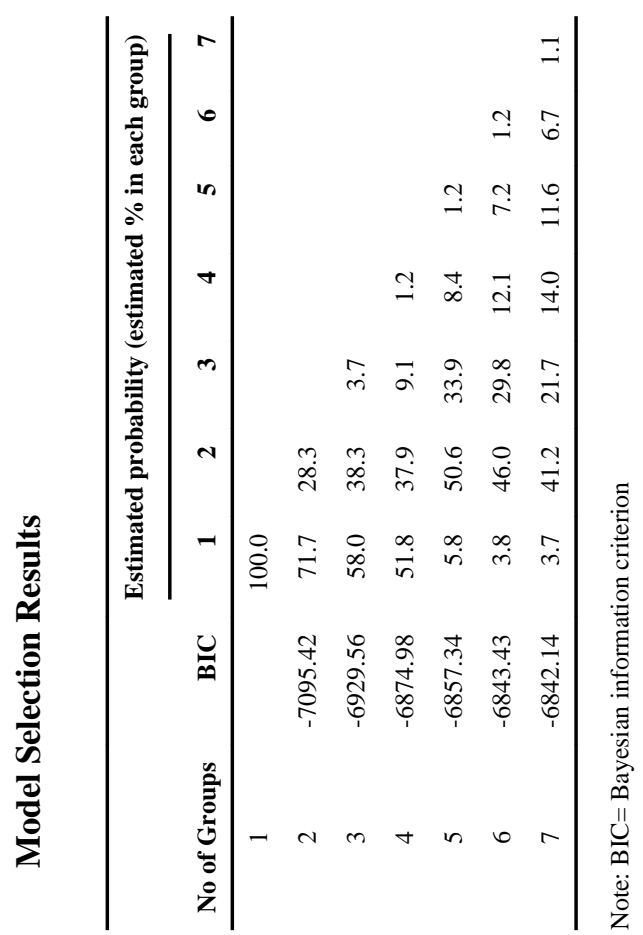

Cancer Epidemiol Biomarkers Prev. Author manuscript; available in PMC 2016 November 01. 


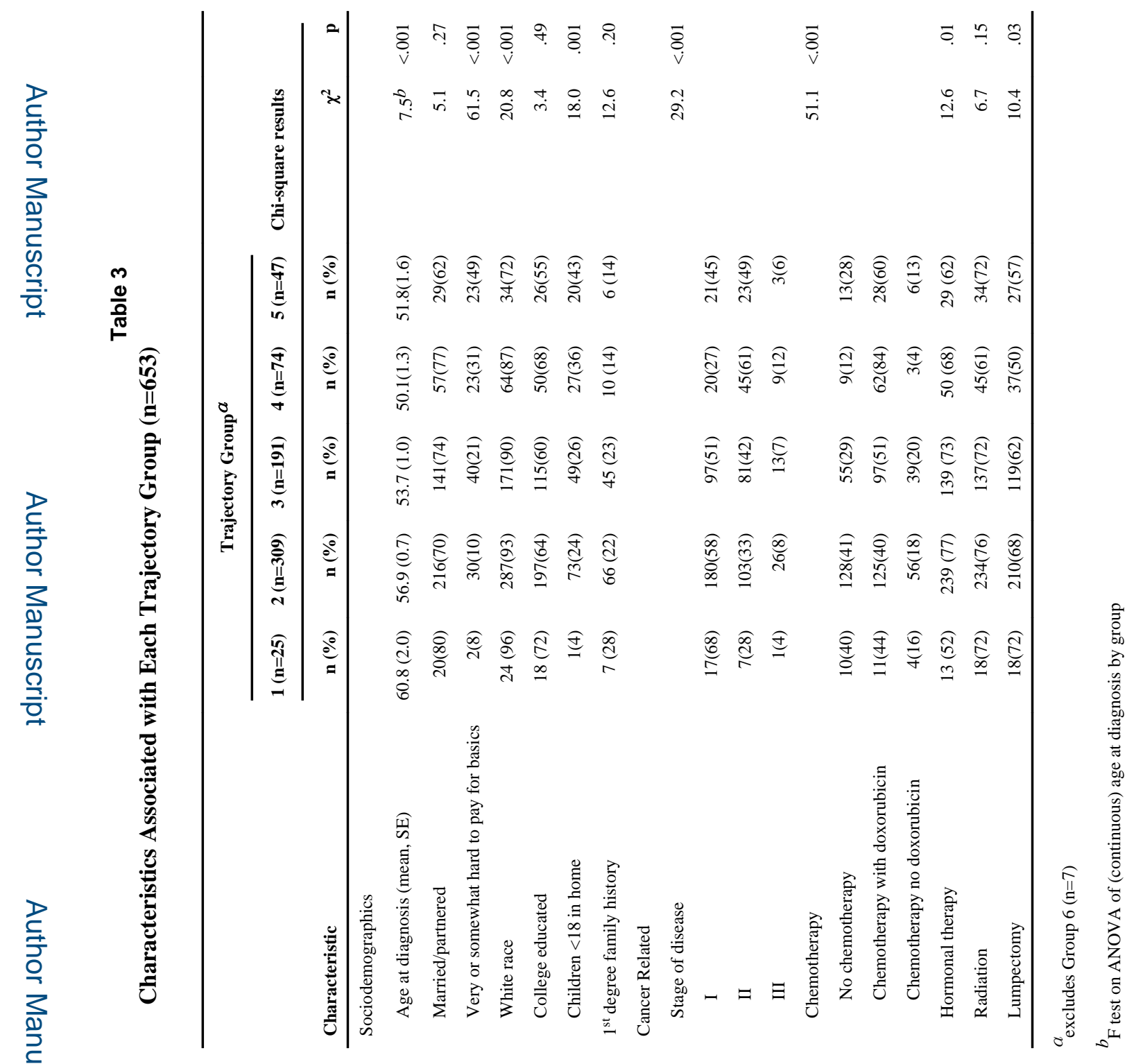




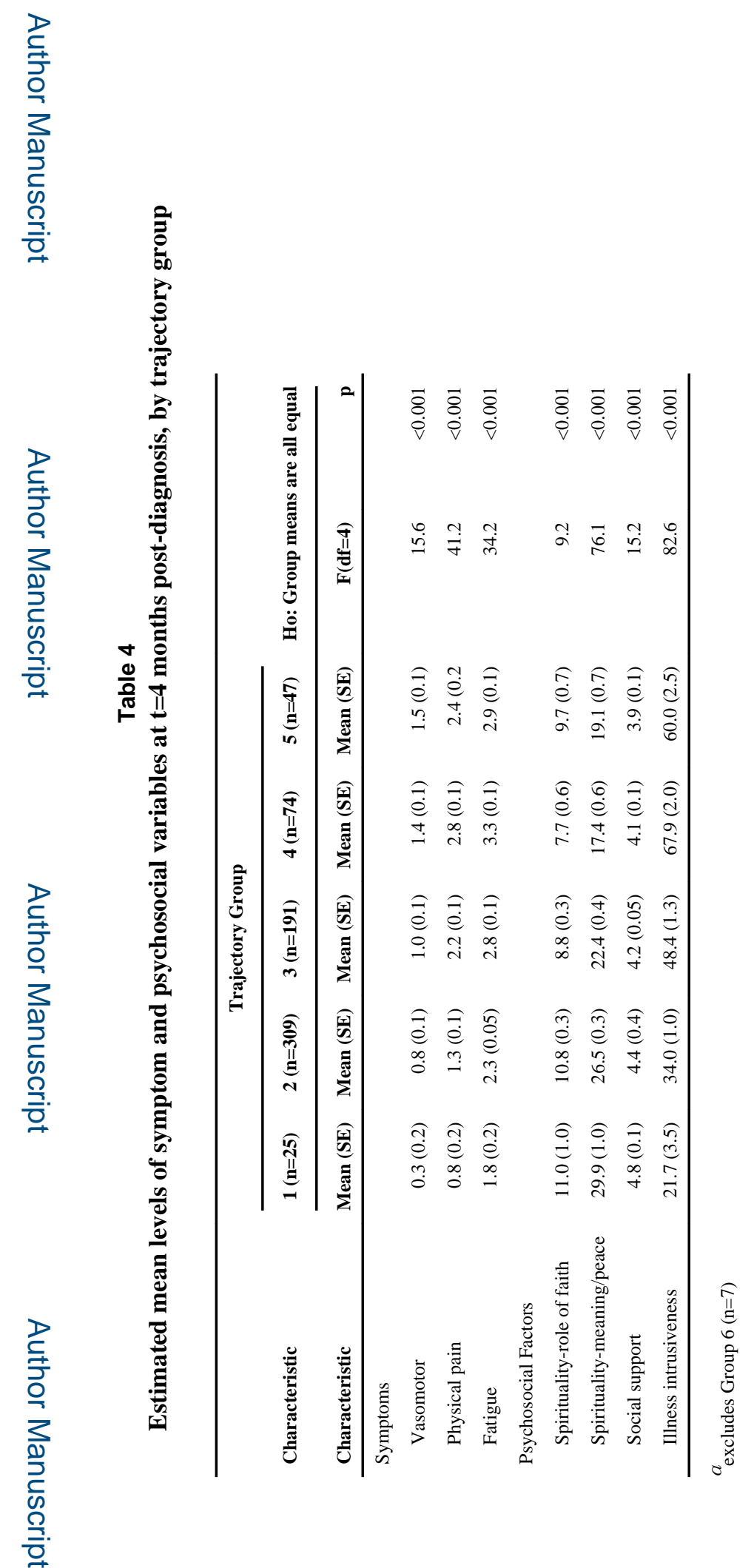

Cancer Epidemiol Biomarkers Prev. Author manuscript; available in PMC 2016 November 01. 